\title{
PROCESSO DE ENFERMAGEM - AVALIAÇÃO FEITA PELOS ALUNOS DO DEPARTAMENTO DE ENFERMAGEM DA UCP *
}

\author{
Rosi Maria Koch ** \\ Luzia Nahoyo Oka
}

\begin{tabular}{l|l|}
\cline { 2 - 2 } & RBEn/06 \\
\hline
\end{tabular}

KOCH, R.M. e OKA, L.N. - Processo de enfermagem - Avaliaçāo feita pelos alunos do departamento de enfermagem da UCP. Rev. Bras. Enf.; DF, 30 : 274-285, 1977.

\section{INTRODUÇÃO}

Em 1975, pela primeira vez em nossa escola, orientamos nossos alunos e aplicamos o Processo de Enfermagem, em duas disciplinas: Enfermagem Médica (alunos do $4 .^{\circ}$ período), e Enfermagem Ginecológica (alunos do $6 .^{\circ}$ período) .

Anteriormente, algumas disciplinas aplicavam só o histórico de enfermagem e o plano de cuidados; outras, apenas o plano de cuidados.

Inicialmente ministramos aulas teóricas sobre a Teoria das Necessidades $\mathrm{Hu}$ manas Básicas e o Processo de Enfermagem, salientando, especialmente, a inıportância de sua aplicabilidade para o desenvolvimento profissional da enfermagem.

Após entrosamento com o pessoal de enfermagem das clínicas, iniciamos a experiência, dando a cada aluno 1 ou 2 pacientes para serem cuidados e fornecemos um roteiro de Histórico de Enfermagem (ANEXO I). Diariamente o aluno deveria preencher a folha de evolução e, através do plano assistencial, traçar e cxecutar o plano de cuidados.

Lendo o artigo de HORTA, resolvemos adaptar o questionário utilizado para avaliar o que nossos alunos perceberam e aproveitaram desta experiência com o Processo de Enfermagem.

* Trabalho apresentado na II Jornada Paranaense de Enfermagem, realizada em Curitiba em maio de 1977.

* Enfermeiras. Professoras do Departamento de Enfermagem da Universidade Católica do Paraná. 
KOCH, R.M. e OKA, L.N. - Processo de enfermagem - Avaliaçāo feita pelos alunos do departamento de enfermagem da UCP. Rev. Bras. Enf.; DF, $30: 274-285,1977$.

\section{DEFINIÇĀO DE TERMOS}

Baseadas na Teoria das Necessidades Humanas Básicas, da Dra. Wanda de Aguiar Horta, temos:

PROCESSO DE ENFERMAGEM: é a dinâmica das açōes sistematizadas e inter-relacionadas, que visa a assistência ao ser humano. Compreende seis fases ou passos: histórico de enfermagem, diagnóstico de enfermagem, plano assistencial, plano de cuidados, evolução e prognóstico de enfermagem.

HISTORICO DE ENFERMAGEM: é o roteiro sistematizado para o levantamento de dados (significativos para a enfermeira) do ser humano, que tornam possível a identificação de seus problemas.

- DIAGNOSTICO DE ENFERMAGEM: é a identificação das necessidades do ser humano que precisam de atendimento, e a determinação, pela enfermeira, do grau de dependência deste atendimento, em natureza e extensão.

PLANO ASSISTENCIAL: é a determinação global da assistência de enfermagem que o ser humano deve receber, face o diagnóstico estabelecido.

- PLANO DE CUIDADOS: é a implementação do plano assistencial, pelo roteiro diário que coordena a ação da equipe de enfermagem na execução de cuidados adequados ao atendimento das nccessidades básicas e específicas do ser humano.

- EVOlUÇÃo DE ENFERMAGEM: é o relato diário das mudanças sucessiva: que ocorrem no ser humano, enquanto estiver sob assistência profissional.
- PROGNOSTICO DE ENFERMAGEM: estimativa da capacidade do ser humano em atender suas necessidades básicas, alteradas após a implementação do plano assistencial e à luz dos dados fornecidos pela evolução de enfermagem.

\section{OBJETIVOS}

1. Indagar a opinião dos estudantes sobre o Processo de Enfermagem;

2. Identificar fatores que interferem na aplicação do Processo de Enfermagem.

\section{METODOLOGIA}

\section{População e amostra}

A população constituiu-se de todos os alunos do $4 .^{\circ}$ e $60^{\circ}$ período do ano letivo de 1975, das disciplinas de Enfermagem Médíca e Enfermagem Ginecológica, respectivamente.

Dos 72 alunos, 25 (34\%) deixaram de preencher o questionário, constituindose a amostra de 47 alunos (66\%).

\section{Instrumento}

Os dados foram colhidos através da aplicação de Questionário (ANEXO II).

\section{Aplicação do instrumento}

O questionário foi distribuido no início do semestre letivo subseqüente ao da aplicação do processo, e recolhido até 2 meses após.

\section{RESULTADOS E DISCUSSAO}

Abaixo transcreveremos os itens constantes do questionário de "Avaliação do Processo de Enfermagem, os respectivos resultados e nossa opinião sobre os dados coletados: 
KOCH, R.M. e OKA, L.N. - Processo de enfermagem - Avaliaçāo feita pelos alunos do departamento de enfermagem da UCP. Rev. Bras. Enf.; DF, 30 : 274-285, 1977.

1. O HISTORICO DE ENFERMAGEM PERMITIU:

\begin{tabular}{lrrrr}
\hline \multicolumn{1}{c}{ ITENS } & \multicolumn{2}{c}{ SIM } & \multicolumn{2}{c}{ NAO } \\
\hline Conhecer profundamente o paciente & N. & $\%$ & N. ${ }^{\circ}$ & $\%$ \\
Individualizar o cuidado & 34 & 72,3 & 13 & 27,7 \\
Planejar o cuidado & 44 & 93,6 & 3 & 6,4 \\
Aprender enfermagem & 47 & 100,0 & - & - \\
Desenvolver observação & 21 & 44,6 & 26 & 55,4 \\
Desenvolver comunicação & 45 & 95,7 & 2 & 4.3 \\
& 39 & 83,0 & 8 & 17,0 \\
\hline
\end{tabular}

Embora 26 alunos $(44,6 \%)$ tenham afirmado que o histórico de enfermagem não serviu para "aprender enfermagem", vemos que a maioria positiva todos os outros itens, o que nos leva a refletir sobre qual o conceito que os alunos têm do que é enfermagem realmente.

\section{COMO VOCE SE SENTIU AO FAZER O HISTORICO DE ENFERMAGEM?}

A maioria dos alunos se sentiu à vontade ou como uma pessoa que quer ajudar, o que é muito bom para o relacionamento enfermeiro-paciente.

Quanto aos $\mathbf{8 , 5 \%}$ que se sentiram constrangidos. ou os $2,1 \%$ que se sentiram como um especulador da vida alheia, vários fatores podem ter concorrido para isso; mas provavelmente o mais evidente é a dificuldade de comunicação. Por outro lado, não deixamos de considerar os problemas referentes ao pouco domínio, por parte do aluno, do conhecimento específico do que é enfermagem, bem como do objetivo do histórico.

\section{COMO OS PACIENTES PARECEM REAGIR DURANTE O HISTÓRICO DE ENFERMAGEM?}

$\mathrm{Na}$ opinião dos alunos, os pacientes, de modo geral, $(57,4 \%)$ ficam muito satisfeitos em ter com quem conversar e a quem contar os problemas, além da vontade de auxiliar o próprio entrevistador.
Quanto aos pacientes que demonstraram inibição para responder o histórico. talvez seja em conseqüência do constrangimento do próprio aluno, que não tem a capacidade de comunicação suficientemente desenvolvida.

4. QUAL SUA OPINIAO SOBRE O HISTORICO DE ENFERMAGEM?

Algumas opiniões favoráveis: 41 (87,2\%)

- "E válido. Mas na prática é quase impossivel devido às condiçōes dos nossos hospitais".

— " " importante porque no histórico levantamos dados básicos sobre o paciente e, com isso, identificamos os seus problemas".

— "É uma forma de explorarmos vários. itens a respeito do indivíduo como pessoa e como paciente".

\section{Opiniões desfavoráveis: $4(8,6 \%)$}

- " $\mathrm{E}$ muito extenso e, por isso, pouco objetivo. Creio que não funciona. Alguns dados são muito pessoais e não levam a nenhum auxílio".

- "Deveriam falar menos dele, simplificá-lo e aplicá-lo mais. Talvez, então, as coisas mudassem".

- "E muito cansativo para o paciente, que não não demonstra muita vontade de contar seus problemas". 
KOCH. R.M. e OKA, L.N. - Processo de enfermagem - Avaliaçāo feita pelos alunos do departamento de enfermagem da UCP. Rev. Bras. Enf.; DF, 30 : 274-285, 1977.

\section{Comentário:}

As opiniōes desfavoráveis ao processo referem-se mais à sua extensão do que à sua eficácia, o que pode ser facilmente corrigido com a aplicação metódica, a valiação, busca de um modelo mais prático, conciso e com o mesmo grau de eficiência.

Nota-se pessimismo por parte de alguns alunos como "creio que não fun. ciona", "na prática é quase impossível nas condiçōes de nossos hospitais". Já estariam os alunos "contaminados" pelo comodismo, pela estrutura obsoleta dos hospitais e em especial dos serviços de enfermagem? Ele, como estudante, realiza. essa atividade. Mas nas clínicas onde estagia, é aplicado o processo?

\section{O DIAGNÓSTICO DE ENFERMAGEM PERMITIU:}

A totalidade das respostas, $100 \%$, demonstrou ter considerado que o diagnóstico de enfermagem permitiu individualizar o cuidado, melhorar o plano assistencial e usar raciocinio lógico, embora $2 ' 7,7 \%$ tenham achado que não serviu para estudar mais enfermagem. Se esse diagnóstico faz com que melhore o planejamento da assistência ao paciente, não serve, também, para aprender mais enfermagem?

Permanece ainda a dúvida do que o aluno considera ser enfermagem como ciência, bem como desconheça, em parte, o seu conceito e o seu conteúdo.

\section{QUAL SUA OPINIAO SOBRE O DIAGNÓSTICO DE ENFERMAGEM?}

Houve um alto percentual de concordância 97,9 - 46 respostas.

Algumas opiniōes:

- "O diagnóstico de enfermagem facilita muito para fazer um bom plano de cuidados e dar um atendimento melhor".

- "Muito importante, porque só assim o paciente é individualizado".

- "Permite conhecimento mais individualizado do paciente e uma avaliação de suas necessidades".

-. "Necessário para partir para o plano assistencial".

7. A ELABORAÇAO DO PLANO ASSistencial E DO PLANO DE CUIDADOS PERMITIU:

\begin{tabular}{|c|c|c|c|c|c|c|}
\hline ITENS & SIM & $\%$ & NĀO & $\%$ & s/resp. & $\%$ \\
\hline $\begin{array}{l}\text { Aprender enfermagem } \\
\text { Melhorar assistência de enfer- }\end{array}$ & 33 & 70,2 & 13 & 27,7 & 1 & 2,1 \\
\hline $\begin{array}{l}\text { magem } \\
\text { Aprender aspectos clínicos da }\end{array}$ & 41 & 87,3 & 6 & 12,7 & - & - \\
\hline $\begin{array}{l}\text { doença } \\
\text { Melhorar cuidados de enferma- }\end{array}$ & 42 & 89,3 & 5 & 10,7 & - & - \\
\hline $\begin{array}{l}\text { gem } \\
\text { Melhorar entrosamento com a }\end{array}$ & 41 & 87,3 & 6 & 12,7 & - & - \\
\hline $\begin{array}{l}\text { equipe eenfermagem } \\
\text { Melhorar entrosamento com a }\end{array}$ & 25 & 53,1 & 21 & 44,8 & 1 & 2,1 \\
\hline $\begin{array}{l}\text { equipe médica } \\
\text { Outros: facilitar entrosamento }\end{array}$ & 23 & 48,9 & 21 & 44,8 & 3 & 6,3 \\
\hline com o paciente & 1 & 2,1 & - & - & - & - \\
\hline
\end{tabular}


KOCH, R.M. e OKA, L.N. - Processo de enfermagem - Avaliação feita pelos alunos do departamento de enfermagem da UCP. Rev. Bras. Enf.; DF, $30: 274-285,1977$.

Os resultados evidenciam quase o mesmo grau de concordância no que se refere à melhoria da assistência de enfermagem,melhoria dos cuidados de enfermagem e aprendizagem dos aspectos clinicos da doença, revelando uma atitude positiva quanto ao papel da enfermagem.

Faltou melhor entrosamento com a equipe médica e mesmo a participação do pessoal de enfermagem, que apenas dieixaram os alunos executarem o processo, sem dele terem participado. Os alunos sentiram isso, pois quase a meta.de da turma $(44,8 \%)$, achou que não melhorou o entrosamento com a equipe médica e de enfermagem. A situação não seria outra se a equipe de enfermagem hospitalar participasse da experiência? Os alunos teriam um modelo a ser seguido e conseqüentemente, sua atuação seria de maior entrosamento.

\section{QUAL A SUA OPINIAO SOBRE O PLANO ASSISTENCIAL E O PLANO DE CUIDADOS?}

Nesta questão foi difícil avaliar quem realmente achou o plano assistencial e o plano de cuidados desfavorável.

Foram consideradas desfavoráveis 19 $(40,4 \%)$ opiniōes que acharam que o plano não é viável ou que não serviu para melhorar a assistência ao paciente. Salientamos que respostas desfavoráveis referiram-se mais a falha da nossa experiência pela dificuldade de execução da assistência planejada.

Acreditamos que, se o planejamento não estivesse sendo executado apenas pelos alunos, mas sim pela equipe hospitalar, a reação seria outra.

\section{Algumas opiniões favoráveis:}

- "E o ideal a ser seguido. Os dois, quando bem elaborados, melhoram sobremaneira 0 trabalho da enfermeira".

- "Auxilia em muitos pontos para o melhor atendimento do paciente $e$ também ajuda todos a participarem"

- "Seria ótimo se fosse feito para todos os pacientes".

\section{Algumas opiniōes desfavoráveis:}

- "A validade do plano asistencial está condicionada à possibilidade de se levar a efeito a assistência programada. Fazer um plano assistencial e deixar o paciente ter alta sem lhe prestar os cuidados é muito negativo".

- "Utopia, enquanto não existir número suficiente de enfermeiras para número de pacientes ou treinar 0 pessoal auxiliar para fazê-lo".

- "E muito complexo. Deveria ser bem mais simplificado para poder ser viável e adaptável à nossa situação".

9. O PREENCHIMENTO DIARIO DA FOLHA DE EVOLUÇAO PERMITIU:

\begin{tabular}{lccccrr}
\multicolumn{1}{c}{ ITENS } & SIM & $\%$ & NAO & $\%$ & s/resp. & $\%$ \\
\hline Observação mais profunda & 45 & 95,8 & 1 & 2,1 & 1 & 2,1 \\
$\begin{array}{l}\text { Melhorar comunicação escrita } \\
\text { Melhorar comunicação oral }\end{array}$ & 42 & 89,3 & 3 & 6.4 & 2 & 4,3 \\
$\begin{array}{l}\text { Conhecimento melhor da } \\
\text { doença }\end{array}$ & 42 & 72,3 & 10 & 21,2 & 3 & 6,5 \\
$\begin{array}{l}\text { Melhor comunicação com o } \\
\text { médico }\end{array}$ & 24 & 51,0 & 21 & 44,7 & 2 & 4,3 \\
$\begin{array}{l}\text { Melhor comunicação com o } \\
\text { paciente }\end{array}$ & 45 & 95,8 & 1 & 2,1 & 1 & 2,1 \\
\hline
\end{tabular}


KOCH, R.M. e OKA, L.N. - Processo de enfermagem - Avaliaçāo feita pelos alunos do departamento de enfermagem da UCP. Rev. Bras. Enf.; DF, $30: 274-285,1977$.

Como não tivemos muito entrosamento com a equipe médica, novamente os alunos sentiram que o preenchimento da folha de evolução não serviu para melhorar a comunicação com o médico $(44,7 \%)$.

Foi alentador verificar que $95,8 \%$ dos alunos acharam que serviu para melhorar a observação, característica imprescindivel do profissional de enfermagem.

\section{QUAL SUA OPINIÃO SOBRE A} FOLHA DE EVOLUÇAO?

Aqui também as opiniōes desfavoráveis $8-17,0 \%$ referiram-se mais às falhas na nossa experiência do que à validade da utilização da folha de evolução.

Algumas opiniões favoráveis:

- "E ótima, pois o paciente apresenta um quadro diferente diariamente $\mathrm{e}$ desse modo podemos acompanhar com mais interesse as mudanças do estado de saúde com involução da doença".

- "E importante porque força a gente a observar mais os pacientes".

- "Permite contato mais direto com o paciente e melhor atendimento".

\section{Algumas opiniōes desfavoráveis:}

- "Na nossa experiência não teve muito valor. Serviu para maior comunicação minha com o paciente. Acho dispensável quando existir relatório de enfermagem na rotina hospitalar".

- "Ainda não atingiu o ponto ideal. A enfermagem precisa orientar o pessoal para observaçōes e anotaçōes mais objetivas".
- " $\mathrm{E}$ necessário entrosamento com a equipe de enfermagem e continuidade nas observaçōes".

11. QUANTO AO PROGNÓSTICO DE ENFERMAGEM, O QUE VOCE ACHA?

HORTA diz que o "prognóstico de enenfermagem é a estimativa da capacidade do ser humano em atender suas necessidades básicas após a implementação do plano assistencial e à luz dos dados fornecidos pela evoluçāo de enfermagem". E pois importante para avalia: a assistência prestada; mas, considerando a estrutura dos nossos serviços, será que ele é essencial? Pelo menos na forna como está sende preconizado $53,2 \%$ opinaram desfavoravelmente e nós pensamos em achar algum meio de verificar onde falhamos (acreditamos ser falta de orientação nossa) ou de testar sua validade real.

\section{Algumas opiniões favoráveis:}

- "Bom, se realmente conseguíssemos fazer aquilo que planejamos".

- "Permite um bom acompanhamento e avaliação do nosso trabalho".

- "Válido, desde que se tenha feito com bastante critério todo o processo de enfermagem".

\section{Algumas opiniōes desfavoráveis:}

— "Não tem aplicação prática".

- "Muito complexo, vago e de pouca validade".

— "Difícil e de pouca validade". 
KOCH, R.M. e OKA, L.N. - Processo de enfermagem - Avaliaçāo feita pelos alunos d. departamento de enfermagem da UCP. Rev. Bras. Enf.; DF, 30 : 274-285, 1977.

12. QUAIS AS MAIORES DIFICULDADES QUEVOCE ENCONTROU AO APLICAR O PROCESSO DE ENFERMAGEM?

I T E N S
N. ${ }^{\circ}$

16

34,0

Falta de trat
assistencial

Falta de tempo para aplicação do processo

Falta de continuidade

10
Dificuldade para expressar com palavras e sentimentos as informaçōes do paciente e os dados observados

Pouca experiência para sua elaboração

Dificuldade para obter os dados do paciente e fazer

exame físico
8

21,2

17,0

10

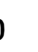

17,0

4

5

4
Se 0 processo de enfermagem fosse aplicado em todos os estágios, dentro de uma linha filosófica ou marco conceptual que reunisse os esforços de todos os docentes, e com melhor entrosamento hospital-escola, provavelmente a maioria das dificuldades seriam abolidas, pois estas resultaram de falta de continuidade e entrosamento com os demais elementos da equipe médica e de enfermagem.

\section{DE SUA OPINIAO SOBRE O PRO- CESSO DE ENFERMAGEM.}

Esta questão, para facilitar a avaliação, foi dividida em tópicos.

Como era uma questão com respostas abertas, é difícil transcrever todas as idéias, mas de modo geral os alunos acharam que:

Para o paciente - o processo de enfermagem ajuda em todos os aspectos, individualizando-o, porque ele se sente mais seguro e tratado como pessoa.
$A$ equipe de enfermagem - acharam que foi difícil a colaboração da equipe, embora se note um melhor entrosamento com aqueles que têm boa vontade.

$A$ equipe médica - não participou muito, talvez mais por não terem conhecimento do assunto do que por resistência à modificação.

Quanto à própria atuação - muito teórico, mas deu para motivar à modificação.

Quanto à viabilidade na prática profissional - infelizmente a maioria não acreditou muito na sua viabilidade, devido principalmente à sua complexidade, exigindo muito tempo para sua execução.

\section{CONSIDERAÇOEES FINAIS}

Muitos enfermeiros que trabalham em escola ou em hospital, relutam em iniciar a aplicação do processo de enfermagem por vários motivos, como: perda do 
KOCH. R.M. e OKA, L.N. - Processo de enfermagem - Avaliação feita pelos alunos do departamento de enfermagem da UCP. Rev. Bras. Enf.; DF, $30: 274-285,1977$.

tempo, falta de pessoal, pouco resultado positivo.

Acreditamos que devemos primeiro testá-lo, para depois falarmos sobre seus defeitos.

E necessário lembrar, ainda, que com a nova legislação sobre o exercício profisisonal, esta será a função específica do enfermeiro em futuro próximo.

Pelos resultados apontados em noss? experiência, concluímos que apesar dc.s dificuldades: falta de entrosamento com a equipe médica e de enfermagem e pouca oportunidade para prestar a assistência planejada, nosso tempo foi muito bem aproveitado e os resultados, tanto para o ensino como para o paciente, foram satisfatórios.
Cremos que, como a aplicação de assistência sistematizada, pode ser diminuído o número de funcionários, e além disso, o enfermeiro aproximando-se mais do paciente, conhecendo-o e planejando seus cuidados, retoma seu lugar de líder da equipe de enfermagem. O tempo que se perde na elaboração do plano e coleta de dados é ganho na prestação de cuidados previamente estabelecidos e adequados.

E de máxima urgência o treinamento de todo o pessoal de enfermagem na área de ensino e de campo de atividades.

Sugerimos que cada profissional dentro de sua área faça uma experiência da aplicação do processo, avalie os resultados e divulgue-os, para que brevemente cheguemos à atuação ideal.

\section{REFERENCIAS BIBLIOGRAFICAS}

HORTA, Wanda de Aguiar - A metodologia do processo de enfermagem. Revisto Brasileira de enfermogem. 24 (6): 81 . 95, out./dez. 1971.

-..- - Enfermagem: teoria, conceitos, prin- cípios e processo. Revista da Esc. Enf. USP, 8 (1): 7-17, mar. 1974.

-...- - Avaliaçāo do histórico de enfermagem pelos estudantes de enfermagem da EEUSP. Revista enfermagem em novas dimensóes, 1 (4): 198-202, 1975. 
KOCH, R.M. e OKA, L.N. - Processo de enfermagem - Avaliaçāo feita pelos alunos do Rev. Bras. Enf.; DF, 30 : 274-285, 1977.

\section{ANEXO I \\ UNIVERSIDADE CATÓLICA DO PARANÁ DEPARTAMENTO DE ENFERMAGEM}

HISTÓRICO DE ENFERMAGEM I

Dadas colhidos por:

Data:

I - IDENTIFICAÇAO:

Nome:

N. ${ }^{\circ}$ Reg.

Enf. .............. Leito ..... Data de Nasc. .../........

Sexo: ......... Cor: ....... Est. Civil: ..... Escolar: .........

Filhos (n.o, idade, sexo)

Religiāo: ..................... Praticante:

Ocupação: ....................... Profissāo:

Naturalidade: ............ Procedência (local, tempo)

Residência temporária:

Responsável: .................... Parentesco:

Endereço para aviso:

Admissão (data, modo)

Diagnóstico médico:

Resumo da doença (dados de literatura):

II - PERCEPÇOES E EXPECTATIVAS:

1 - Por que procurou o hospital?

2 - Como foi recebido?

3 - A que atribui a doença atual?

4 - O que sabe sobre a sua doença?

5 - O que sabe sobre o seu tratamento?

6 - O que o preocupa?

7 - O que pensa de anestesia e operação? Tem medo?

8 - O que acha de ficar junto com outras pessoas no quarto?

9 - Espera receber visitas? De quem?

10 - Quais seus planos para quando sair do hospital?

III - EXPERIENCIAS ANTERIORES COM DOENÇA E HOSPITAL:

1 - Quantas vezes e onde já esteve internado?

2 - Que impressōes guardou destas internaçōes?

3 - O que espera deste hospital?

IV - MEIO AMBIENTE - (procedência)

1 - Habitação (tipo da casa, n. ${ }^{\circ}$ cômodos, n.o pessoas, utensílios) 
KOCH, R.M. e OKA, L.N. - Processo de enfermagem - Avaliaçāo feita pelos alunos do departamento de enfermagem da UCP. Rev. Bras. Enf.; DF', $30: 274-285,1977$.

2 - Agua (procedência, Itipo)

3 - Sanitário (localização, tịpo)

4 - Lixo (tratamento)

5 - Animais (cães, gatos, moscas, mosquitos, barbeiro, galinha, porco)

V - IMUNIZAÇOES:

1 - Que vacinas tomou? Quando?

VI - HABITOS:

1 - Alimentação

- alimentos preferidos:

- alimentação mais comum:

- n. ${ }^{\circ}$ de refeiçōes ao dia e horário:

- apetite ................ Mastigação:

- digestão ................. Náuseas

- líquidos (volume diário)

2 - Eliminação intestinal

- freqüência:

- características (consistência, ar, odor, dor)

Constipação: ........ Diarréia: ........ Flatulência:

O que usa para tratar constipação?

O que usa para tratar diarréia?

$O$ que usa para gases?

3 - Eliminação urinária

- freqüência:

Tem algum problema? (dor. ardor, prurido, sangramento...)

Características (ar, odor, sedimento)

4 - Menstruação

- Ciclo .............. quantidade, duração:

Problemas (dismenorréia, amenorréia, etc.)

Costuma lavar a cabeça quando menstruada?

Usa anticoncepcional?

5 - Higiene

1 - Banho: Como toma banho?

Com que freqüência?

Aparência: 
KOCH. R.M. e OKA, L.N. - Processo de enfermagem - Avaliaçāo feita pelos alunos do departamento de enfermagem da UCP. Rev. Bras. Enf.; DF, $30: 274-285,1977$.

2 - Boca: Escova dentes? Quando? Com quê? Tem dor de dente? Usa prótese? Como Cuida?

3 - Unhas: Costuma cortar? Quando? Exame (aparência)

4 - Cabelos: Quando lava? Com quê? Como penteia?

5 - Tricotomia: Costuma fazer? Usa desodorante?? Talco?

6 - Sono e recreação:

1 - Quantas horas costuma dormir?

2 - Insônia? O que faz para dormir?

3 - Como costuma passar o tempo de folga? (rádio, TV, leitura, clube, jogos)

7 - Fumo:

1 - viciado? Quantos por dia? ..........

2 - Tem problema respiratório?

8 - Alcool:
1 - Costuma beber bebidas alcoólicas?
Quanto?
Já bebeu mais antes?
O quê?

VII - ALERGIAS:

Já apresentou reação alérgica a alguma coisa ou remédio?

VIII - QUEIXAS:

Quais suas dores?

O que costuma fazer para tratá-las?

IX - EXAME FISICO:

1 - Estado geral (constituição física, aspecto)

2 - Estado mental

3 - Locomoção

4 - Postura

5 - Vestuário

6 - Sinais vitais: Tem.: ....... Pulso: ....... Resp.: ....... P.A.: ............ Peso:

7 - Pele (integridade, aspecto)

8 - Cabeça (condiçōes, limpeza)

9 - Ouvido (acuidade auditiva) 
KOCH, R.M. e OKA, L.N. - Processo de enfermagem - Avaliaçáo feita pelos alunos do departamento de enfermagem da UCP. Rev. Bras. Enf.; DF, $30: 274-285,1977$.

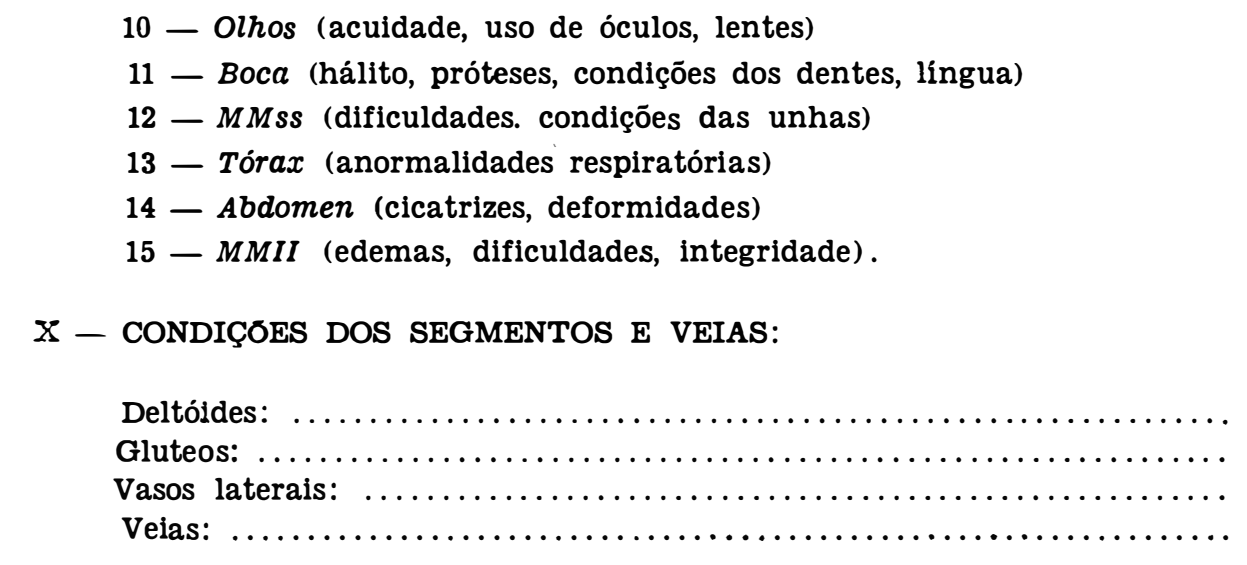

XI - DADOS MEDICOS DE INTERESSE PARA A ENFERMAGEM: (pode obter do prontuário)

1 - História da doença:

2 - Exames de laboratório:

Data Tipo Resultado

3 - Resumo sucinto de cirurgia:

4 - Prescrição médica:

XII - AJUSTAMENTO AO HOSPITAL:

1 - Quais suas queixas quanto ao hospital?

2 - O que acha de seus colegas de enfermaria?

3 - O que gostaria de fazer no hospital?

4 - O que gostaria de perguntar?

XIII - IMPRESSOES DO ENTREVISTADOR: 